\title{
The development of a nurse-led iron deficiency anaemia service in a district general hospital
}

\author{
Susan L Surgenor, Silvia Kirkham, Sally D Parry, Elizabeth J Williams, \\ Jonathon A Snook
}

Gastroenterology Unit, Poole Hospital NHS Foundation Trust, Poole, UK

\section{Correspondence to}

Dr Jonathon A Snook, Gastroenterology Unit, Poole Hospital NHS Foundation Trust, Longfleet Road, Poole, Dorset BH15 2JB, UK; Jonathon. Snook@gmail.com

Received 2 September 2013 Revised 15 October 2013 Accepted 16 October 2013 Published Online First

17 December 2013
To cite: Surgenor $\mathrm{SL}$, Kirkham S, Parry SD, et al. Frontline Gastroenterology 2014;5:219-223.

\begin{abstract}
Objective To improve the quality of care provided to patients with iron deficiency anaemia (IDA).

Design Service development.

Setting District General Hospital.

Patients Adults with IDA.

Main outcome measures Descriptive report of the practicalities and benefits of establishing an

IDA clinic.

Conclusions The IDA clinic is a novel service development which enhances the management of patients with this common condition, by facilitating prompt confirmation of the diagnosis, replacement therapy and investigation for serious underlying pathology, in particular gastrointestinal malignancy.
\end{abstract}

\section{INTRODUCTION}

Iron deficiency anaemia (IDA) is a common clinical problem. ${ }^{1-4}$ The overall incidence exceeds one case per 1000 population per annum, and in the elderly the figure is considerably higher. ${ }^{5}$ IDA is frequently diagnosed in the primary care setting, but is also seen across a range of specialties in secondary care, resulting in considerable variation in management standards.

The importance of establishing and acting on the diagnosis of IDA is twofold. First, while IDA is the cause of considerable morbidity, it is eminently treatable. Second, about a third of cases in men and postmenopausal women will have significant underlying gastrointestinal (GI) pathology, often in the absence of localising symptoms-and a third of these will have GI malignancy. ${ }^{1-5}$

\section{THE PROBLEM}

Despite the prevalence of IDA-and the importance of IDA as an early indicator of underlying pathology-it is sometimes rather poorly managed. Our impression is that this remains the case despite clear and concise national guidelines (recently updated $^{4}$ ). Our original observations indicated issues at a number of levels, which included:

1. Anaemia not recognised as being due to iron deficiency on the one hand (particularly when the mean red cell volume (MCV) was normal), or incorrectly attributed to iron deficiency on the other. An inhouse audit in 2003 showed that $13 \%$ of referrals with what was thought to be IDA were not, in fact, truly iron deficient.

2. Inappropriate treatment by oral iron replacement without further investigation into the cause, or by emergency admission for blood transfusion.

3. Delayed and/or incomplete investigation for underlying GI pathology. For example, an audit in 2003 showed that only $73 \%$ had a small bowel biopsy to exclude coeliac disease, and that $40 \%$ of patients waited more than 8 weeks for their GI investigations.

4. Inappropriate referral for endoscopic investigation of patients with major comorbidities and/or frailty.

5. Inadequate follow-up to confirm a sustained haematological response to iron replacement.

6. Failure to act on the results of investigations into the cause of IDA.

\section{TOWARDS A SOLUTION}

To address these multiple issues, we established a specialist nurse-led IDA clinic in Poole in 2004, run under the auspices of two consultant gastroenterologists. Referral numbers have risen progressively, reaching over 200 in 2012. 
To accommodate these numbers and consequent follow-ups, there are currently two clinics a week.

Referrals to the IDA clinic are accepted from primary and secondary care, as long as evidence of iron deficiency has been provided-by means of either iron studies, or demonstration of a convincing haematological response to iron supplementation. A simplified version of the algorithm followed by the IDA clinic is shown in figure 1.

At the initial clinic visit, the diagnosis of underlying iron deficiency is verified, and a urine sample is checked for evidence of haematuria, which might indicate a cause for IDA in the renal tract. The necessary patient data is collected efficiently by means of an IDA-specific proforma (figure 2)-this informs the patient register, which is important for follow-up purposes as well as service audit and observational research. ${ }^{5}$ Iron replacement therapy is initiated, with consideration given to intravenous iron infusion in patients who have particularly profound anaemia.

The rationale for GI investigation is discussed, along with the practicalities-in the light of comorbidities, previous investigations and patient preference. Premenopausal women are seen in the clinic if referred, but such cases are counselled regarding the low yield of GI investigation in this patient group, and gynaecological referral is encouraged if there is clear evidence of menorrhagia. Individuals with evidence of iron deficiency but without anaemia are also seen, but again counselled regarding the relatively low yield of investigation. The merits of investigation of borderline cases are discussed with the

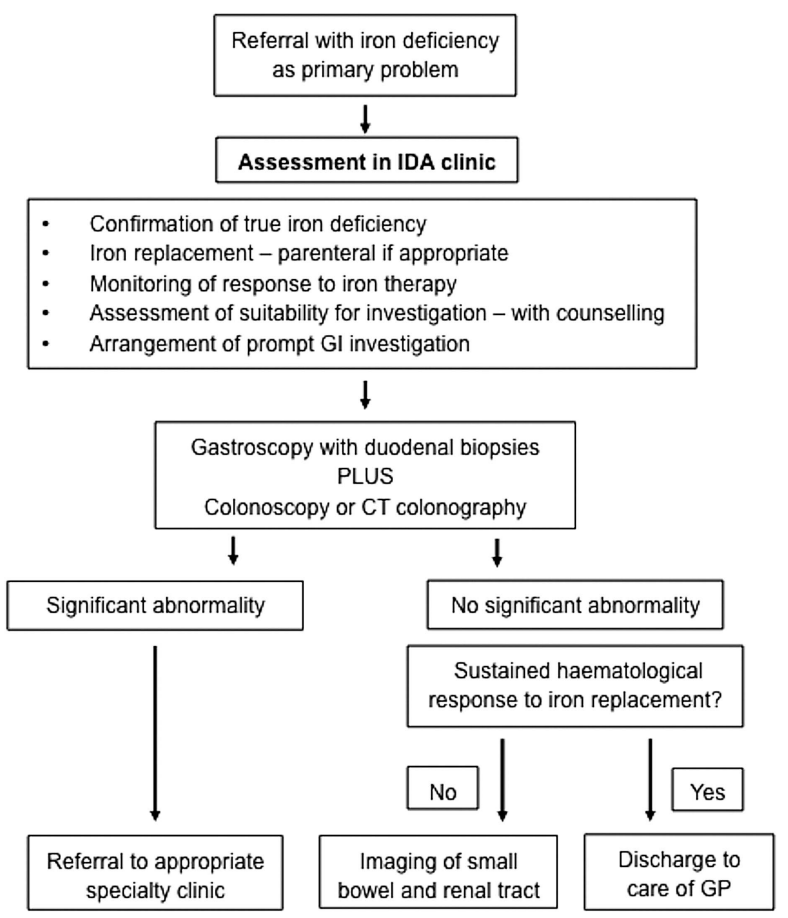

Figure 1 Simplified algorithm followed by the iron deficiency anaemia clinic. overseeing consultant gastroenterologist and the patient concerned.

The IDA clinic team arranges fast-track GI investigations-generally combined gastroscopy with small bowel biopsy and colonoscopy (bidirectional endoscopy (BDE)) - and checks regularly for results. Patients who prove to have significant pathology are referred directly on to the appropriate specialty area. The paired paper ${ }^{5}$ provides details of the prevalence of significant GI pathology in our IDA cohort, and an analysis of the clinical indicators of underlying malignancy.

About $70 \%$ of patients have no significant abnormalities on examination of the upper and lower GI tract. Most in this group exhibit a complete haematological response following a pulse of iron replacement therapy that is sustained on a follow-up blood test at 3 months. These patients are discharged, as our experience is consistent with the literature indicating that the likelihood of undiagnosed underlying pathology is low. ${ }^{6}$

Iron deficiency proves to be persistent or recurrent following replacement therapy in a minority of patients with negative BDE. These patients are investigated further to exclude, in particular, underlying blood loss from either the small bowel or renal tract. Many individuals in this small subgroup prove to have pathology in the small bowel on capsule endoscopymost commonly, vascular malformations.

\section{COST AND BENEFITS}

The running costs of the IDA clinic are relatively small, and relate primarily to specialist nurse salaries with secretarial support for two clinics per week. This is more than offset by savings, relating, in particular, to the reduced usage of blood transfusion (see below). There are, however, considerable additional benefits. First, the clinic facilitates rapid access—addressing the national guideline that patients with IDA should be assessed within 2 weeks of referral (see audit data below).

Second, the clinic identifies at an early stage those individuals in whom further investigation for iron deficiency may not be indicated. This includes those who (1) are not truly iron deficient, (2) have recently undergone adequate investigation of their GI tract, or (3) have major comorbidities that might preclude safe endoscopy. Such patients are managed as appropriate.

Third, the clinic provides an opportunity for counselling referrals regarding the rationale for-and the process of $-\mathrm{BDE}$, ensuring that an early slot is offered if the patient decides to proceed. A small but significant minority of patients elect not to. Audits before and shortly after introduction of the IDA clinic revealed a rise in the percentage of subjects investigated within 6 weeks of the date on the referral letter from $43 \%$ to $79 \%$ (figure 3 ). The figure has risen further since then. 

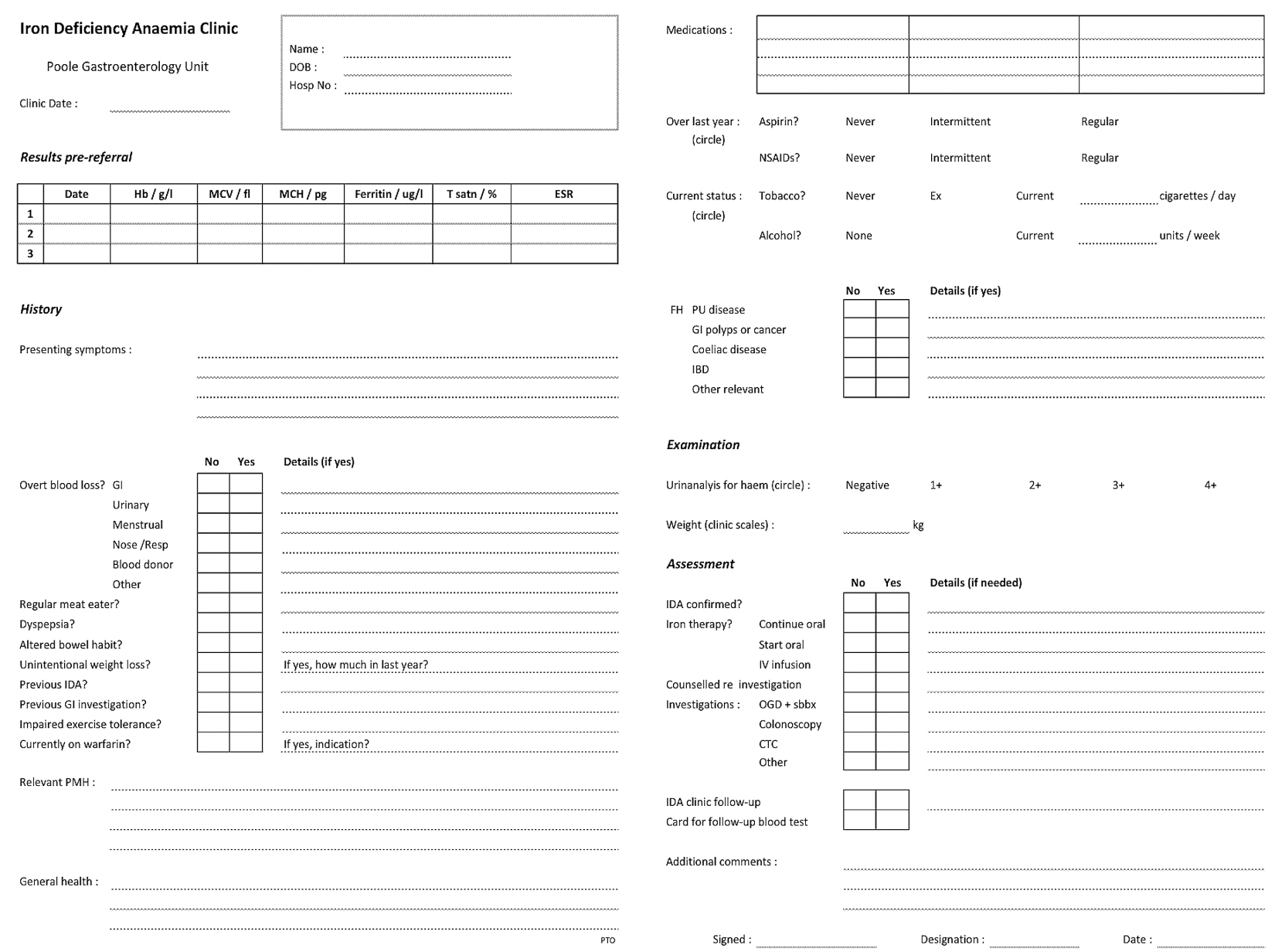

Figure 2 The Poole iron deficiency anaemia clinic new patient assessment proforma.

Fourth, the deficiency is corrected appropriately. This is generally achieved with oral iron supplementation, but prompt intravenous total dose iron infusion is arranged-as a safer and more cost-effective alternative to blood transfusion-if the anaemia is particularly profound and/or symptomatic, or if imminent surgery is anticipated. ${ }^{7} 8$ In our experience, initial haemoglobin recovery rates of up to $25 \mathrm{~g} / \mathrm{L}$ per week can be achieved following total dose iron infusion.

There has been a substantial fall over the last decade in the number of patients admitted to Poole Hospital each year for blood transfusion to treat IDA, and this mirrors the rise in the number of patients seen in the IDA clinic (figure 4). While there may of course have been other contributory factors, we suspect that development of the IDA clinic has-directly or indirectlybeen the major reason for this reduction in blood usage. The cost equation is complex, but assuming an average of 3 units per transfusion, and a cost of $£ 130$ per unit, this equates to savings of approximately $£ 30000$ per annum in the use of blood alone.

Finally, the clinic team ensures that postendoscopy follow-up is timely and appropriate to the diagnosisfor example, bowel cancer in the colorectal surgery clinic, coeliac disease in the coeliac clinic. Interval blood tests are arranged, and the minority of cases with negative BDE who do not exhibit a full and sustained response to iron replacement therapy are considered for imaging of the small bowel and renal tract in particular.

\section{AUDIT}

A recent audit of all patients seen in the IDA clinic during the course of $2012(n=230)$ revealed that $73 \%$ were seen within 2 weeks of referral. The commonest reason for failure to meet this target was the delay in primary care referrals reaching the hospital that resulted from submission to the local 'Choose \& Book' facility rather than via the 'two week wait' route.

Following assessment, $86 \%$ underwent BDE, while $8 \%$ had more limited endoscopic investigation for a variety of reasons, most commonly an expressed preference for minimal-preparation CT colonography rather than colonoscopy. The remaining $6 \%$ did not proceed with further investigation on the grounds of patient preference, major comorbidity, or frailty.

An iron infusion was administered to $12 \%$ of the audit cohort. Of those with no significant abnormalities on initial GI examination, 25 (20\%) exhibited 


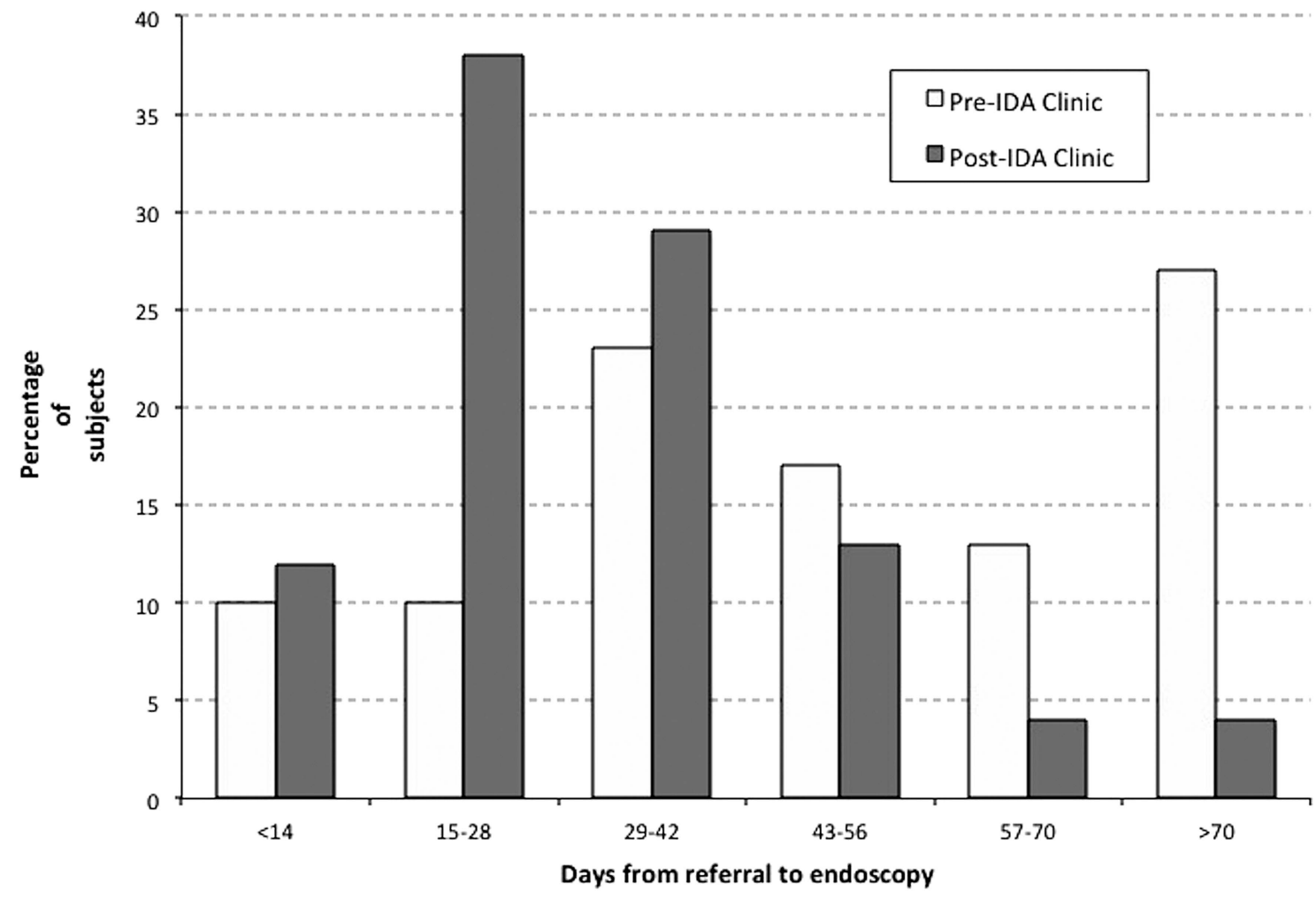

Figure 3 Comparison of the delay from referral to endoscopy for subjects with confirmed iron deficiency anaemia, before and after introduction of the Poole iron deficiency anaemia clinic in 2004.

persistent or recurrent IDA, and went on to have further investigation as appropriate to the individual case. This revealed a variety of diagnoses, including (1) renal carcinoma and (2) metastatic melanoma. Over $50 \%$ of the 12 cases who went on to have capsule endoscopy proved to have pathology in the small bowel, notably multiple vascular malformations (6), and Crohn's disease (1).

\section{DISCUSSION}

IDA is, in a sense, an 'orphan' condition, in that it is not always perceived to fall comfortably within the remit of one particular specialty. In view of the prevalence of underlying GI pathology and the accepted requirement for gastrointestinal investigation in many cases, we would argue that IDA is best managed under the auspices of the gastroenterology service. The IDA clinic effectively provides this facility.

There are several reasons why IDA without localising symptoms lends itself to management in a nurse-led, problem-orientated clinic. First, it is a relatively homogeneous condition, yet one that may initially present to a range of disciplines across primary and secondary care. Second, optimal management requires the application of a fairly straightforward algorithm (figure 1). Finally, counselling and assiduous follow-up are important aspects of care.

Our previous experience of slow and incomplete investigation of IDA is well described in the literature.
A large cohort study from Birmingham found that only $35 \%$ of 578 patients with IDA who subsequently proved to have colorectal cancer were initially referred to an appropriate specialty with a delay of many months before establishing the underlying diagnosis in some cases. ${ }^{9}$ On the other hand, a retrospective study from Lewisham revealed that an astonishing 56\% of secondary care endoscopy referrals for anaemia did not have evidence of iron deficiency, at considerable cost in terms of wasted resources. ${ }^{10}$

The Poole IDA clinic is a novel development that is proving popular with local medical teams, and the progressive rise in referral numbers from primary and secondary care is testament to this. It is equally popular with patients, and we now rarely receive complaints regarding inadequate assessment or investigation. We also believe that the clinic may indirectly provide an educational service on the grounds that while the total clinic referral numbers are progressively rising, the proportion with adequate confirmation of true iron deficiency is climbing in parallel.

We have seen an impressive fall in the number of admissions with IDA on the medical take for blood transfusion since the IDA clinic was established. Iron infusions are a more logical treatment for iron deficiency than blood transfusion, and provide more sustained control of anaemia in the longer term. ${ }^{78}$ They are also less expensive and safer than transfusion, and do not require overnight admission to administer. 


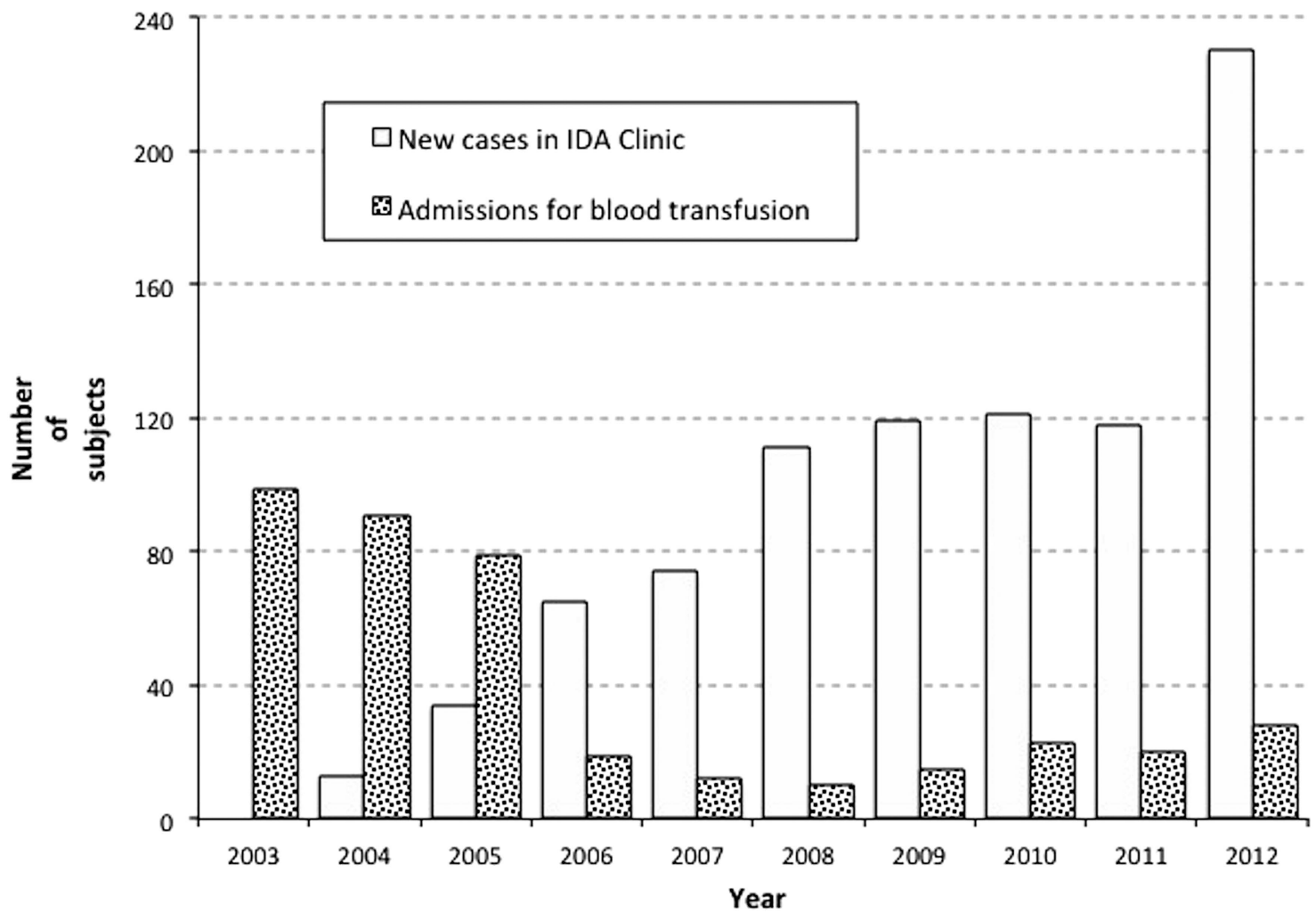

Figure 4 The number of subjects by year (1) assessed in the iron deficiency anaemia clinic and (2) admitted (overnight or day-case) for blood transfusion to treat iron deficiency anaemia.

In conclusion, our experience over the last 9 years is that the IDA clinic is a novel development that rationalises the assessment, investigation and treatment of patients with IDA. It also accelerates the process of particular relevance to the $10 \%$ of subjects with underlying malignancy. Finally, it improves the use of resources, in particular, by reducing the (ab)use of blood transfusion, and the number of inappropriate referrals for endoscopic investigation.

Acknowledgements We are indebted to Christine Stewart, Senior Information Analyst, Poole Hospital NHSFT, for the provision of the hospital statistics data shown in figure 4.

Contributors SLS, EJW and JAS conceived and designed this report. SLS and SK collected the data and drafted the initial manuscript, while JAS was responsible for data collation. All authors made significant contributions to the subsequent revision of the paper, and have approved the final version prior to submission.

Competing interests SLS and EJW have received honoraria for speaking at educational meetings sponsored by Pharmacosmos.

Provenance and peer review Not commissioned; externally peer reviewed.

\section{REFERENCES}

1 Liu K, Kaffes AJ. Iron deficiency anaemia: a review of diagnosis, investigation and management. Eur J Gastroenterol Hepatol 2012;24:109-16.
2 Pasricha SR, Flecknoe-Brown SC, Allen KJ, et al. Diagnosis and management of iron deficiency anaemia: a clinical update. Med J Aust 2010;193:525-32.

3 Zhu A, Kaneshiro M, Kaunitz JD. Evaluation and treatment of iron deficiency anemia: a gastroenterological perspective. Dig Dis Sci 2010;55:548-59.

4 Goddard AF, James MW, McIntyre AS, et al. Guidelines for the management of iron deficiency anaemia. Gut 2011;60:1309-16.

5 Castro-Silva A, Sheppard ZA, Surgenor SL, et al. Clinical risk factors for underlying gastrointestinal malignancy in iron deficiency anaemia. In press. doi: 10.1136/flgastro-2013-100386

6 Sahay R, Scott BB. Iron deficiency anaemia-how far to investigate? Gut 1993;34:1427-8.

7 Gozzard D. When is high-dose intravenous iron repletion needed? Assessing new treatment options. Drug Des Dev Ther 2011;5:51-60.

8 Jahn MR, Andreasen HB, Futterer S, et al. A comparative study of the physicochemical properties of iron isomaltoside 1000 (Monofer), a new intravenous iron preparation and its clinical implications. Eur J Pharm Biopharm 2011;78:480-91.

9 Damery S, Ryan R, Wilson S, et al. Iron deficiency anaemia and delayed diagnosis of colorectal cancer: a retrospective cohort study. Colorectal Dis 2011;13:e53-60.

10 Mankodi S, Hayee BH, O’Donohue J, et al. Anaemia investigation in practice: inappropriate, cost inefficient with a risk of missing gastrointestinal cancer. Can we improve? Clin Med 2010;10:115-18. 